\title{
Deconstruyendo el resultado contable convencional para diseñar un resultado contable ambiental
}

\author{
Deconstructing the conventional accounting result to design an \\ environmental accounting result
} José Juan Déniz Mayor* y María Concepción Verona Martel

Universidad de Las Palmas de Gran Canaria, Campus de Tafira, España

Recibido el 7 de abril de 2014; aceptado el 24 de agosto de 2014

Disponible en Internet el 2 de julio de 2015

\section{Resumen}

En el presente trabajo se discute la dificultad de delimitar con precisión los flujos de gastos e ingresos relacionados con la gestión ambiental corporativa, utilizando como hilo argumental el diseño de un modelo algebraico de resultado contable que haga visible las partidas más significativas en esta materia. Las principales conclusiones se centran en la necesidad de vincular los gastos e ingresos ambientales a objetivos específicos a fin de poder evaluar el desempeño ambiental de la empresa y el riesgo de adoptar decisiones ambientalmente ineficientes para mejorar la imagen pública gracias a este estado contable. Entre las principales limitaciones del modelo propuesto se encuentran el problema de la vinculación homogeneizada de las magnitudes contables a los objetivos de protección ambiental y la exclusión de las externalidades ambientales.

Derechos Reservados (C) 2015 Universidad Nacional Autónoma de México, Facultad de Contaduría y Administración. Este es un artículo de acceso abierto distribuido bajo los términos de la Licencia Creative Commons CC BY-NC-ND 4.0.

Palabras clave: Ingresos ambientales; Gastos ambientales; Modelo algebraico; Resultado contable ambiental; Contabilidad ambiental
Abstract
In this paper, the difficulty of accurately defining the flows of expenses and income related to corporate environmental management, using as storyline the design of an algebraic model of accounting result to

\footnotetext{
* Autor para correspondencia.

Correo electrónico: josejuan.deniz@ulpgc.es (J.J. Déniz Mayor).

La revisión por pares es responsabilidad de la Universidad Nacional Autónoma de México.
} 
make visible the most significant items in this area, is discussed. The main conclusions are centred in the need to link environmental protection expenditures and environmental revenues to specific goals in order to assess the company environmental performance and the risk of taking environmentally inefficient decisions to improve the public image thanks to this statement. The main limitations of the proposed model are the problem of linking accounting homogenized variables to environmental protection goals and the exclusion of environmental externalities.

All Rights Reserved (? 2015 Universidad Nacional Autónoma de México, Facultad de Contaduría y Administración. This is an open access item distributed under the Creative Commons CC License BY-NC-ND 4.0.

Keywords: Environmental income; Environmental expenses; Algebraic model; Environmental income result; Environmental accounting

\section{Introducción}

En el contexto de sus procesos decisorios, los usuarios de la información contable empresarial se apoyan, entre otros estados contables, en la cuenta de resultados del ejercicio, la cual recoge, de forma debidamente ordenada y agregada, de acuerdo con determinados criterios, los diferentes flujos de gastos e ingresos habidos como consecuencia de la actividad llevada a cabo por la empresa.

A este respecto, puede suceder que determinadas consecuencias del desempeño ambiental corporativo, en concreto las derivadas no solo de los esfuerzos llevados a cabo para prevenir, corregir o minimizar el impacto ambiental causado por las operaciones de la empresa, sino también, y en su caso, de la apropiación ambientalmente adversa de los bienes y servicios ofrecidos por el entorno, permanezcan invisibles a los ojos de los grupos de interés al no figurar de forma explícita los gastos e ingresos asociados en los estados contables, obteniéndose una visión no realista acerca de la gestión en la materia.

Bien es cierto que los estados financieros al uso (balance, cuenta de resultados, estado de cambios en el patrimonio neto, estado de flujos de efectivo y notas a los estados financieros) no están diseñados para abarcar el extenso espectro de relaciones entre la empresa y su medio natural, salvo que las mismas puedan ser objeto de reconocimiento contable de acuerdo con el contexto político-legal, económico y social, teniendo presentes los criterios convencionalmente admitidos (responder a una definición de elemento, bien sea como activo, pasivo, patrimonio neto, gasto o ingreso; poseer una valoración fiable; y ser probable la generación o, en su caso, salida de recursos que incorporan beneficios económicos).

Evidentemente, una ampliación del objeto material de la contabilidad conlleva cuestionarse la delimitación de la frontera organizativa y operacional. La hipótesis de entidad contable, es decir, el postular la existencia de una unidad económica diferenciada del resto, dentro de cuyos límites se define un área de interés y unos eventos concretos sobre los que informar, está sujeta a una continua revisión. Así, frente a la noción clásica, que parte de la consideración de una unidad económica responsable de ciertas actividades y su control administrativo, existe una acepción más amplia que tiene presentes los intereses económicos de los partícipes, de tal forma que son las necesidades informativas de estos últimos, y no las actividades desarrolladas por la entidad, las que definen los límites de la entidad contable (Belkaoui, 1995).

Aunque la opinión dominante se apoya en la acepción clásica, esto es, considerar como sujeto contable el conjunto de entidades vinculadas sometidas a su control administrativo, existen eventos 
que aunque se originan en el seno de dichas entidades, sus efectos se manifiestan fuera de su círculo, bien en otras personas, bien en otras empresas o entidades, o en el propio medio ambiente. Es el caso de las actividades externalizadas (operaciones que son realizadas por terceros en lugar de la propia empresa al objeto de deslocalizar riesgos y responsabilidades, aunque constituyan el núcleo del negocio) ${ }^{1}$ y de las externalidades ambientales negativas (hechos que se producen cuando una actividad ocasiona un impacto sobre el medio ambiente, los seres humanos, sus propiedades y su bienestar, sin existir una obligación legal o contractual por parte del causante de resarcir o compensar a las partes afectadas).

No obstante lo expuesto, y sin abandonar el marco conceptual estándar, es factible reclasificar y agregar, a efectos informativos, los elementos contables asociados a la gestión ambiental, que (es oportuno aclarar) ya están reconocidos por el sistema de información contable de la entidad, si bien de forma indiferenciada. De entre tales elementos, son los gastos e ingresos, convenientemente clasificados, los que permitirían conformar una magnitud agregada denominada resultado contable ambiental, como exponente de la renta neta generada por la empresa como consecuencia de su impacto sobre el medio ambiente.

Debe aclararse que el término «ambiental» es profusamente utilizado en los pronunciamientos en materia contable para referirse al reconocimiento, medida y valoración de las actuaciones llevadas a cabo por la empresa para prevenir, corregir o minimizar los efectos ocasionados por sus operaciones sobre el entorno, no siendo equivalente al término «sostenible», pues solo cabría hablar de sostenibilidad si se da el salto hacia la inclusión de los costos y beneficios de las externalidades tanto económicas como sociales y ambientales, en el cómputo del resultado contable, cuestión que no es abordada en este trabajo.

Desde la perspectiva expuesta, la empresa podría comenzar con la presentación de forma diferenciada de los gastos defensivos incurridos, lo cual, por otra parte, ya se viene haciendo de forma habitual en diferentes sectores económicos, bien de forma voluntaria, bien por imperativo legal. La siguiente etapa podría consistir en mostrar los ingresos vinculados tanto al así llamado «negocio ambiental» como a las operaciones ordinarias del sistema de gestión ambiental, de manera que los grupos de interés pudieran percibir que las actividades realizadas en dicho campo también pueden tener un «benéfico influjo» en la cuenta de resultados. Una fase más avanzada podría ser el reconocimiento explícito de aquellos gastos e ingresos relacionados con transacciones perjudiciales para el medio ambiente, de manera que pudieran identificarse no solo los aspectos positivos de la gestión ambiental, sino también los negativos. No obstante, esta opción es poco probable que se acometa dada la escasa predisposición de las empresas a comunicar malas noticias (Gray, Bebbington y Walters, 1993; Bennett y James, 1997) y su especial preocupación por la reputación (Michael, 2003; Newell, 2005; Cho, Roberts y Patten, 2010) a costa de camuflar la insostenibilidad de sus actuaciones (Moneva, Archel y Correa, 2006).

El objetivo del presente trabajo es construir un modelo algebraico de resultado contable ambiental, entendido como agregado de los flujos de gastos e ingresos de tal carácter relacionados con las actividades corporativas ordinarias. El orden expositivo es el siguiente: en la siguiente sección

\footnotetext{
${ }^{1}$ En algunos estudios se sostiene que las empresas ubican las actividades con efectos ambientales más nocivos en aquellos países que gozan de una legislación ambiental más laxa, limitándose a adaptar sus políticas de gestión a las exigencias legales del país (Leonard, 1988; Martínez Alier, 1992; Stewart, 1993) o, incluso, llegando a presionar a la baja en salarios y estándares sociales (Gladwin, Kennelly y Krause, 1995; Greider, 1997). Graves accidentes industriales, como los acaecidos en una empresa química en Bhopal (India) en 1984 y una fábrica textil en Savar (Bangladesh) en 2013, parecen abonar esta presunción.
} 
se estudiarán los gastos considerados positivos para el medio ambiente, mientras que en el tercer apartado se incluirán, además, los ingresos ambientalmente positivos, destinándose el cuarto bloque a introducir la problemática de los gastos e ingresos negativos para el entorno. El trabajo finaliza con las principales conclusiones y consideraciones, y una relación de la bibliografía citada.

\section{El resultado ambiental como agregado de gastos ambientales positivos}

Una primera aproximación al cálculo del resultado ambiental consiste en ceñir su cómputo a los gastos incurridos por la organización, o en nombre de esta, en actividades de prevención, corrección o minimización de daños al medio ambiente, es decir, los denominados gastos ambientales o, más concretamente y en atención al objetivo del presente trabajo, gastos ambientales positivos. Ahora bien, la empresa puede incurrir en gastos de diversa índole que pueden presentar como característica distintiva su vinculación a hechos de carácter ambiental, si bien puede afirmarse que la simple delimitación de lo que constituye un gasto ambiental no es tarea fácil, ya que incluso la propia noción de gasto defensivo, muy común en el campo de la Contabilidad Nacional, está abierta a discusión, dada su ambigüedad, pues como señala Jaszi (1973), «los gastos de alojamiento protegen del frío y de la lluvia [...], los gastos médicos protegen de la enfermedad, y los desembolsos por motivos religiosos, de las llamas del infierno».

En este sentido, Eurostat (2007, p. 58), tras establecer una definición de «actividades» indicando que son todo aquello que «tiene lugar cuando recursos tales como equipos, mano de obra, técnicas de producción, redes de información o productos son combinados para crear bienes o servicios específicos», señala que se considerarán a efectos estadísticos como actividades de protección ambiental «las actividades (que incluyen equipos, mano de obra, técnicas de producción, redes de información o productos) cuyo propósito principal sea recolectar, tratar, reducir, prevenir o eliminar contaminantes y la contaminación o cualquier otra degradación del medio ambiente debido a la presión de las actividades humanas» ${ }^{2}$.

Es interesante la matización expuesta en torno a la noción de «propósito principal». Así, la intención perseguida al realizar una actividad puede ser diferente en función de los actores participantes y sus fines estratégicos. A título ilustrativo, el objetivo de adoptar medidas de eficiencia energética por parte de una empresa industrial puede ser la reducción de costos de aprovisionamiento de electricidad y combustibles, y no tanto mitigar su contribución al cambio climático; sin embargo, para una administración pública la realización de campañas de ahorro energético puede perseguir fundamentalmente un propósito ambiental como la reducción del impacto sobre el clima y, de forma secundaria, disminuir la dependencia de fuentes de energía extranjeras.

Debe tenerse presente que la dirección de la compañía, en determinados casos y guiada por un propósito manipulativo frente a los grupos de interés, podría caer en la tentación de asignar el calificativo de ambientales a partidas contables que realmente no lo son, o que siéndolas respondan a una realidad distinta a la que se desea transmitir. Por tal motivo, deben establecerse unos criterios precisos de delimitación de lo que es un gasto ambiental, como los señalados por la Comisión Europea (2001, anexo 2.2), que lo define como «el coste de las medidas adoptadas por una empresa, o por otras en nombre de esta, para evitar, reducir o reparar daños al medio ambiente que resulten de sus actividades ordinarias», o el Instituto de Contabilidad y Auditoría

${ }^{2}$ El subrayado aparece en el texto original. 
Tabla 1

Ejemplos de gastos ambientales ordinarios

Gastos de personal dedicado a tiempo parcial o en exclusiva a las actividades ambientales (entre las que se incluirían la construcción de activos ambientales)

Gastos de formación

Servicios médicos (prevención, seguimiento y tratamiento) relacionados

Mantenimiento del sistema de información ambiental

Consumo de energía y otros suministros relacionados con la gestión ambiental (por ejemplo, consumibles para

depuradoras, repuestos, fungibles para mediciones, etc.)

Amortizaciones de activos ambientales

Tasas y autorizaciones que no tengan carácter plurianual

Servicios exteriores (por ejemplo, consultorías ambientales, transporte de residuos, etc.)

Costos por cierre de la actividad, así como los derivados del mantenimiento y control de las instalaciones una vez desmanteladas

Gastos financieros incurridos para la adquisición de activos ambientales

Sobrecostos por la adquisición de suministros menos contaminantes que sus equivalentes convencionales

Recogida, tratamiento y eliminación de residuos (incluyendo sistemas integrados de gestión)

Etc.

Fuente: Elaboración propia.

de Cuentas (2002, norma 4.1) español, que lo circunscribe a «los importes devengados, de las actividades medioambientales realizadas o que deban realizarse, para la gestión de los efectos medioambientales de las operaciones de la entidad, así como los derivados de los compromisos medioambientales del sujeto contable. Entre ellos se sitúan los gastos ocasionados por la prevención de la contaminación relacionada con las actividades operativas actuales, el tratamiento de residuos y vertidos, la descontaminación, la restauración, la gestión medioambiental o la auditoría medioambiental».

De acuerdo con lo expuesto, la magnitud resultante estaría integrada, en una primera aproximación, por conceptos tales como los señalados en la tabla $1^{3}$.

En general, todas las definiciones consideran que la calificación del gasto como ambiental no se halla tanto en su naturaleza como en su función, con lo cual, conceptos como los salarios no constituyen un gasto ambiental hasta que se produce su aplicación a las actividades ambientales de la empresa, en la fracción que corresponda en el caso de que los servicios prestados tuviesen un carácter parcialmente ambiental (Comisión Europea, 2001, anexo 2.2; Instituto de Contabilidad y Auditoría de Cuentas, 2002, norma 4.3).

Considerando lo expuesto, cabría distinguir entre gastos considerados ambientalmente positivos, por razones legales, contractuales o por asunción voluntaria por la entidad $(G p)$, y otros gastos de la entidad $(G o)$, obteniéndose la siguiente expresión analítica representativa del gasto imputable al ejercicio $(G)$ :

$$
G=G p+G o
$$

Los gastos ambientales positivos pueden ser objeto de desglose en atención a la dimensión que se desee analizar (factor ambiental afectado, objetivos operativos, naturaleza del gasto, función

\footnotetext{
${ }^{3}$ La relación no es exhaustiva. En la página de Internet del Instituto Nacional de Estadística español puede encontrarse una enumeración detallada de actividades y gastos de protección del medio ambiente (http://www.ine.es/jaxi/menu.do?type=pcaxis\&path=\%2Ft26\%2Fp070\&file=inebase \&L=0). Asimismo, la norma $U N E$ 50011:2005 Gestión ambiental. Guía para la evaluación de los costes ambientales. Costes ambientales internos incluye su propia clasificación orientativa (Asociación Española de Normalización y Certificación, 2005).
} 
Tabla 2

Clasificación del gasto en función del factor ambiental

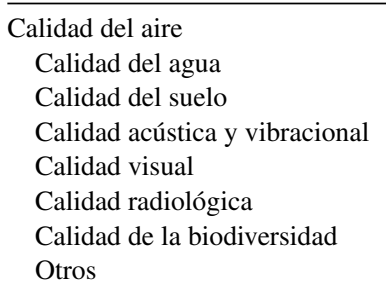

Fuente: Elaboración propia.

del gasto o sección o departamento en que se aplican). Así, para cada criterio clasificatorio $c$ habría un gasto ambiental diferenciado $G p_{c}$, pudiendo reformularse la expresión (1) como:

$$
G=\sum_{c=1}^{z} G p_{c}+G o
$$

En las tablas 2-6 se exponen algunas posibles tipificaciones de gastos considerando la dimensión analizada.

Según lo expuesto, podría obtenerse una medida del resultado contable $(R)$ como la que se expone a continuación, en la que $I$ representa el valor de los ingresos correspondientes al ejercicio:

$$
R=I-(G p+G o)
$$

Un refinamiento de este enfoque consistiría en clasificar las magnitudes ambientales desde la perspectiva de los modelos causales. Es el caso del modelo Presión-Estado-Respuesta, propuesto por la Organisation for Economic Co-operation and Development $(1993,1997,2003)$ a partir del estudio de Friend y Rapport (1979) y usado como guía para los informes nacionales sobre el estado del medio ambiente en países miembros de dicha organización, así como para la formulación de indicadores de desarrollo sostenible en la realización de proyectos por la CEPAL (Guttman Sterimberg, Zorro Sánchez, Cuervo de Forero y Ramírez, 2004; Quiroga Martínez, 2007).

Tabla 3

Clasificación del gasto ambiental atendiendo a objetivos operativos o actividades

Protección del aire ambiente y el clima

Gestión de aguas residuales

Manejo de residuos

Protección y remediación de suelos, aguas subterráneas

Mitigación de ruido y vibraciones

Protección de la biodiversidad biológica y del paisaje

Protección de radiaciones

Investigación y desarrollo

Otras actividades de protección ambiental

Administración general del medio ambiente

Educación, capacitación e información

Actividades que generan gastos indivisibles

Actividades no especificadas en otra parte

Fuente: Eurostat (2000). 
Tabla 4

Clasificación del gasto ambiental considerando su naturaleza

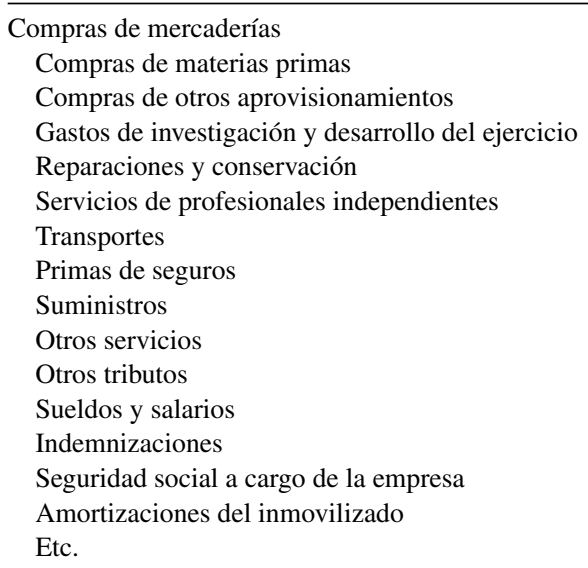

Fuente: Elaboración propia.

Tabla 5

Clasificación del gasto ambiental atendiendo a la función desempeñada

Prevención
Control y mitigación
Conservación
Restauración
Medición y vigilancia
Planificación
Administrativos

Fuente: Elaboración propia.

Existen variantes como el modelo Presión-Estado-Respuesta/Efectos (United States Environmental Protection Agency, 1995) o el modelo Fuerzas inductoras-Estado-Respuesta (United Nations, 1995; United States Environmental Protection Agency, 1995; Mortensen, 1997). Este último enfoque parte de la noción básica de que existen determinadas fuerzas inductoras (por ejemplo, las necesidades de los consumidores o la agenda política) que ejercen presiones (tales como emisiones contaminantes o cambios en el uso del suelo) sobre el medio ambiente, las cuales inducen cambios en el estado del medio (por ejemplo, alteraciones en los niveles de contaminantes ambientales, la biodiversidad, los cursos de agua, etc.). Ante estos

Tabla 6

Clasificación del gasto ambiental en función del departamento principal
Aprovisionamiento
Producción
Comercialización
Administración
Etc.

Fuente: Elaboración propia. 


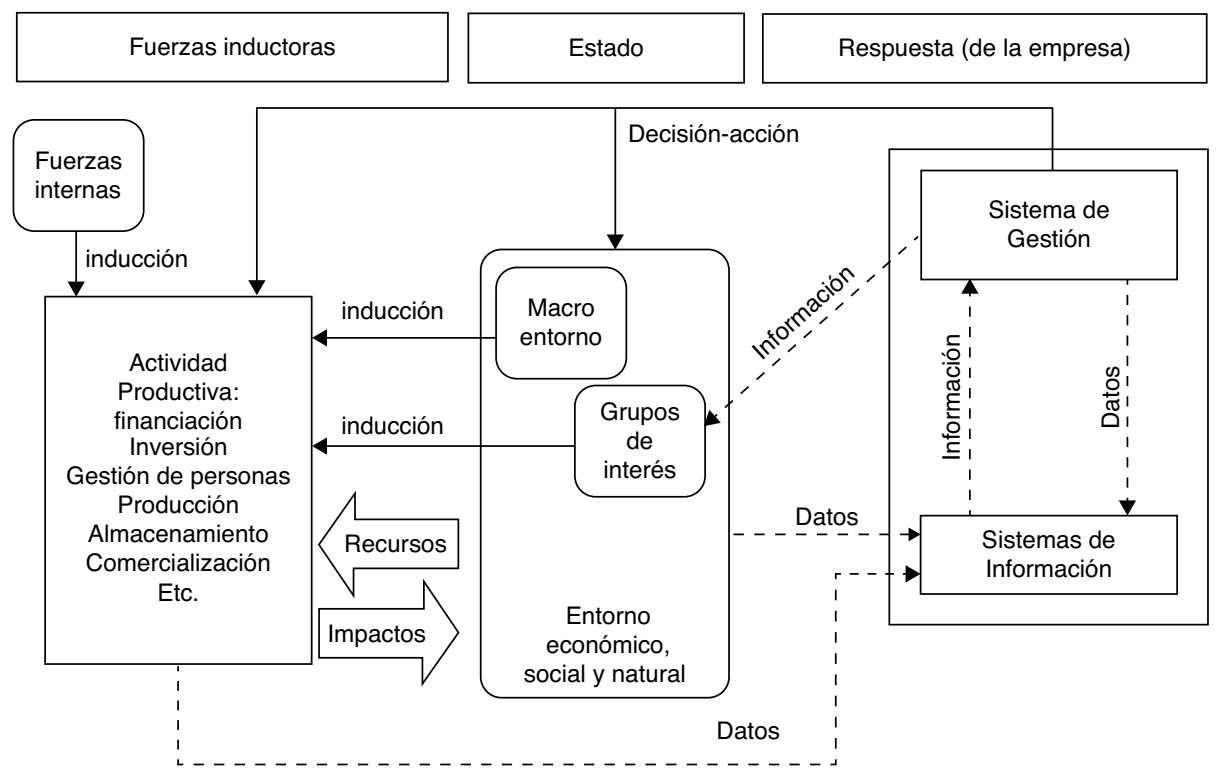

Figura 1. Adaptación del modelo Fuerzas inductoras-Estado-Respuesta a la gestión empresarial. Fuente: Déniz Mayor y Verona Martel (2012).

hechos, la sociedad responde con actuaciones políticas, así como con programas económicos y ambientales al objeto de prevenir, reducir o mitigar las presiones causantes y/o el daño causado al entorno.

De acuerdo con la versión propuesta por Déniz Mayor y Verona Martel (2012) para la gestión empresarial (esquematizada en la fig. 1), las actuaciones de prevención, corrección y minimización ambiental constituyen respuestas de la organización a las variables de presión vinculadas a su actividad empresarial y, en su caso, a los impactos causados sobre las variables de estado ambiental. Un reparto de gastos de acuerdo con este enfoque permitiría conocer la cuantía de los recursos asignados a cada variable ambiental y su relevancia, al menos desde el punto de vista monetario.

Dado que unos altos niveles de gasto ambiental no tienen por qué estar necesariamente correlacionados con una buena gestión ambiental per se (Atkinson, 2000), surge la necesidad de establecer determinados indicadores de control y seguimiento. La compañía puede trabajar con magnitudes presupuestadas o bien con comparaciones con otras empresas del sector (benchmark), por lo que el resultado contable ambiental también podría configurarse como un instrumento de control de gestión, a través del cual se comparasen previsiones o importes sectoriales (debidamente ponderados, en su caso) de gasto ambiental positivo $\left(G p^{p}\right)$ con gastos ambientales positivos realizados o ejecutados por la entidad $\left(G p^{r}\right)$. Su saldo, que podría catalogarse como desviación de gastos ambientales positivos $(D g p)$, constituiría el montante de las diferencias producidas:

$$
D g p=G p^{p}-G p^{r}
$$


Tabla 7

Gastos de imagen y relaciones

Participación voluntaria en actividades comunitarias de protección del medio

Creación, edición y distribución del informe ambiental anual

Análisis de la demanda de productos amigables con el entorno

Realización de estudios sobre posibilidades futuras de suministro de materiales sensibles y posibilidades de sustitución

Identificación de prácticas similares en competidores (benchmarking)

Donaciones para fines ambientales

Etc.

Fuente: Elaboración propia.

Adicionalmente, si se desea una mayor precisión en la evaluación del desempeño, se podrían descomponer los gastos para cada tipo de variable de estado $i\left(G p_{i}\right)$ o presión $j\left(G p_{j}\right)$ :

$$
D g p=\sum_{i=1}^{n}\left(G p_{i}^{p}-G p_{i}^{r}\right)+\sum_{j=1}^{m}\left(G p_{j}^{p}-G p_{j}^{r}\right)
$$

Asimismo, se pueden establecer objetivos de cumplimiento ambiental distinguiendo entre objetivos ambientales positivos previstos (propios o a partir de un benchmark del sector, convenientemente cuantificados) $\left(O^{p}\right)$ y objetivos ambientales realizados o ejecutados por la entidad $\left(O^{r}\right)$, que, ponderados por los gastos ambientales previstos o de benchmark y los gastos efectivamente realizados, determinaría un índice de eficiencia ambiental (Iea) en el logro de dichos objetivos:

$$
\text { Iea }=\frac{O^{p}}{G p^{p}}-\frac{O^{r}}{G p^{r}}
$$

Si se desea establecer indicadores de objetivos y gastos para cada tipo de variable de estado $i$ ( $O_{i}$ y $G p_{i}$, respectivamente) o presión $j$ ( $O_{j}$ y $G p_{j}$, respectivamente), ello permitiría diseñar sistemas de indicadores que mostrasen el grado de eficiencia en la gestión del medio ambiente, como el que se expone a título ilustrativo:

$$
\text { Iea }=\sum_{i=1}^{n}\left(\frac{O_{i}^{p}}{G p_{i}^{p}}-\frac{O_{i}^{r}}{G p_{i}^{r}}\right)+\sum_{j=1}^{m}\left(\frac{O_{j}^{p}}{G p_{j}^{p}}-\frac{O_{j}^{r}}{G p_{j}^{r}}\right)
$$

La consideración de un gasto como ambientalmente positivo se sustenta normalmente en una relación de causalidad directa entre el daño y las actividades llevadas a cabo para su prevención y/o corrección. Esta atribución, apoyada en la visión reduccionista de la regulación contable vigente, centrada en los gastos defensivos, supone generalmente la exclusión de las actividades de imagen y relaciones. Estas partidas presentan como principal problema la dificultad de determinar los beneficios ambientales asociados, sobre todo cuando son utilizadas como medio para manipular las percepciones del público, desviando la atención de la compañía hacia sus diversas actividades filantrópicas y de patrocinio ambiental. Dichas clases de gasto podrían ser objeto de un adecuado reconocimiento o discriminación, aunque de forma diferenciada respecto de los gastos defensivos, al no implicar un efecto directamente mensurable en el estado del medio ambiente y las presiones sobre el entorno. A modo de ejemplo, cabe citar las partidas indicadas en la tabla 7.

En este sentido, es factible cuestionarse si la consideración de gasto ambiental debe circunscribirse única y exclusivamente a los gastos defensivos o si debería ampliarse la definición para 
abarcar todos los gastos incurridos por la organización que produzcan, directa o indirectamente, un impacto positivo, mediato o inmediato, cierto o probable sobre el estado del medio ambiente. Por otra parte, la importancia de los gastos menos tangibles va a depender, entre otros extremos, de la posición competitiva de la compañía: si se halla en un entorno fuertemente competitivo, los gastos de imagen corporativa pueden ser esenciales para su supervivencia. Sin embargo, en otras compañías que ocupen una posición relevante en el mercado y carezcan de presiones externas, puede que tales gastos sean menos importantes, orientándose básicamente al cumplimiento de regulaciones y la satisfacción de sus accionistas.

Esta visión ampliada trataría de tomar en consideración todas las posibles conexiones e interacciones entre la entidad y el medio ambiente, aunque dentro de los límites de un criterio de razonabilidad basado en la existencia de una relación contrastada entre el hecho causante (la transacción económica a considerar) y su impacto físico sobre las variables de presión y/o estado ambiental. De esta forma, los agentes económicos podrían formarse un juicio racional y adoptar los cursos de acción que se consideren oportunos en el marco de la política ambiental de la entidad y las expectativas de los grupos de interés, siendo conscientes de que, dada su complejidad, puede no existir una relación biyectiva entre causa y efecto. Es decir, puede existir una causa y varios efectos o un efecto y varias causas.

Teniendo en cuenta lo expuesto, la expresión representativa del gasto, reflejada en (1), podría ser modificada para mostrar los gastos de imagen y relaciones (Gir), identificados de entre el resto de los gastos de la empresa $(G o)$ :

$$
G=G p+G i r+G o
$$

Mención especial cabe realizar de las indemnizaciones y sanciones, así como de las dotaciones a provisiones por responsabilidad ambiental. En el primer caso, tales partidas están constituidas por las penalizaciones legales y las demandas por daños causados a empleados o ciudadanos en general, así como a la propiedad o al medio ambiente sujeto a protección legal. Por su parte, las provisiones por responsabilidad ambiental tratan de hacer frente a obligaciones legales, contractuales o voluntariamente asumidas por la empresa para prevenir o reparar daños al medio ambiente o bien a terceros y sus propiedades, sean accidentales o intencionados, debido a un incumplimiento de sus obligaciones por parte de la entidad, no siendo compensados por otras vías $^{4}$.

Estos conceptos, si bien responden a la idea de resarcir o compensar al entorno, las personas y las propiedades por los daños sufridos, son consecuencia de la elusión por parte de la entidad de sus compromisos de prevenir, corregir o reducir el daño ambiental, de ahí que, a pesar de representar un impacto curativo, deban ser representados de forma diferenciada. Si se analizan las definiciones de gasto ambiental propuestas por organismos como Eurostat (2000), la Comisión Europea (2001) o, a nivel español, el Instituto Nacional de Estadística (2013), las partidas alusivas a indemnizaciones y sanciones son excluidas de las mismas. Así, el Instituto de Contabilidad y Auditoría de Cuentas (2002) español señala en su norma 4.4 que «[s]e calificarán como gastos extraordinarios aquellos que se produzcan como consecuencia de hechos acaecidos fuera de la

\footnotetext{
${ }^{4}$ Cuestión diferente es la relativa a los costos de desmantelamiento, retiro o rehabilitación del inmovilizado. En este caso, la empresa incurre en dichas obligaciones en el momento de adquirir el inmovilizado o para poder utilizar el mismo durante un período de tiempo determinado. De acuerdo con la regulación vigente internacional (International Accounting Standards Board, 2003, 16, par. 16c; International Accounting Standards Committee, 1998, 37, par. 45), se consideran un mayor precio de adquisición o coste de producción del activo y no un gasto, siendo ajustado su importe por las actualizaciones de valor que vayan surgiendo hasta el final de las operaciones con el elemento.
} 
actividad ordinaria de la entidad y se espere que no se produzcan con frecuencia. Entre estos gastos extraordinarios se incluyen las multas, las sanciones y las compensaciones a terceros por perjuicios causados por los daños medioambientales».

La razón de ello podría hallarse en el intento de evitar transmitir al público una percepción errónea acerca del comportamiento ambiental corporativo. Una empresa con un fuerte historial de sanciones por daño ambiental podría presentar debidamente maquillados los desembolsos realizados por tal concepto como indicador de una buena gestión en favor de la protección del medio natural. Prueba de ello es que en los años ochenta entre un 30 y un $60 \%$ de todo el gasto declarado por las empresas estadounidenses como «conservación del medio ambiente» consistiese en multas y sanciones (Silverstein, 1989).

La identificación y medida de los gastos ocasionados por el mal desempeño en materia de medio ambiente, puesto de manifiesto a través de las indemnizaciones, sanciones y dotaciones de pasivos (Gis), que se escindirían de la magnitud alusiva a otros gastos (Go), llevaría a la modificación de la expresión representativa del gasto $(G)$, reflejada en (8), para diferenciar este tipo de hechos contables:

$$
G=G p+G i r+G i s+G o
$$

Teniendo en cuenta lo expuesto, el resultado reflejado en (3) presentaría, además y debidamente desglosados, los gastos de imagen y relaciones y los gastos ocasionados por indemnizaciones, sanciones y dotaciones de pasivos ambientales:

$$
R=I-(G p+G i r+G i s+G o)
$$

\section{La identificación de ingresos ambientales positivos}

Determinar qué debe entenderse por ingreso ambiental positivo constituye una problemática más compleja, si cabe, que la delimitación del concepto de gasto ambiental positivo. En la literatura se observa una escasa referencia a la noción de ingreso ambiental, por considerar posiblemente más interesantes los gastos ambientales y su gestión. No obstante, parece detectarse una vinculación de dicho término con el producto de la venta de bienes y servicios suministrados por las empresas que participan en el denominado negocio ambiental. Así, Organisation for Economic Co-operation and Development/Eurostat (1999, p. 9) definen dicho concepto como «la actividad que produce bienes y servicios para medir, prevenir, limitar, minimizar o corregir daños ambientales en el agua, el aire y el suelo, así como problemas relacionados con residuos, ruido y ecosistemas. Se incluyen las tecnologías limpias, productos y/o servicios que reducen los riesgos medioambientales, que minimizan la contaminación y el uso de recursos».

A título ilustrativo, en la tabla 8 se presenta una relación exhaustiva de ingresos relacionados con el negocio ambiental reconocidos como tales por el Instituto Nacional de Estadística (2013) español. Una versión más detallada puede encontrarse en Eurostat (2009).

Esta caracterización tiene su lógica dado que tanto el Instituto Nacional de Estadística como Eurostat operan en términos de contabilidad nacional: es decir, si a la hora de definir los gastos ambientales se incluyen como tales determinadas adquisiciones de bienes y servicios, es razonable que, a su vez, se consideren como ambientales determinadas ventas de bienes y servicios concordantes con los primeros.

Además de la relación expuesta cabría considerar como ingresos ambientales, entre otros, los derivados de las ventas de bienes destinados a su reciclaje o reutilización, o la concesión de licencias de uso de tecnologías limpias. También podrían incluirse las subvenciones y donaciones 
Tabla 8

Bienes y servicios considerados como producción ambiental

Electrodomésticos y aparatos domésticos de la categoría más eficiente en sus categorías (por ejemplo, etiquetado A++); otros productos con la etiqueta energética de la UE en la categoría más alta de eficiencia (A)

Vehículos: fabricación de vehículos eléctricos

Productos biodegradables: producción de detergentes y jabones; bolsas hechas de bioplásticos; pinturas al agua Biocombustibles, biodiésel y biogás: producción

Eficiencia energética: lámparas fluorescentes compactas y lámparas de descarga; calentadores de agua solares; termostatos para la regulación de temperatura; válvulas termostáticas

Ecoetiqueta: productos con etiqueta ecológica de la UE o ecoetiqueta

Aislamiento: fabricación de productos y materiales para aislamiento térmico o acústico: ventanas de alto rendimiento energético; paneles de vidrio de hojas múltiples para aislamiento; corcho, lana de madera, lana de roca; fibra de vidrio; materiales de celulosa, poliuretano o polímeros de estireno en formas primarias

Filtros y emisiones: producción de carbón activado para filtros; fabricación de maquinaria o componentes para la filtración de líquidos y gases con finalidad medioambiental; filtros textiles para la reducción de emisiones y para tratamiento de aguas residuales; fabricación de silenciadores y tubos de escape Instrumentos de medida: instrumentos fabricados con una clara finalidad medioambiental

Reciclaje: fabricación de equipamiento para reciclaje de vidrio, papel, plástico, madera; neumáticos recauchutados y caucho regenerado; construcción de plantas de reciclaje; fabricación de esos productos reciclados; fabricación de otros productos con utilización de esos productos reciclados

Desalinizadoras: producción de agua desalinizada; construcción de plantas desalinizadoras; fabricación de equipamiento para plantas

Energías renovables: producción de energía renovable; construcción de plantas y fabricación de equipos para la producción; servicios de ingeniería para la producción; fabricación de paneles solares, turbinas eólicas e hidráulicas y sus componentes

Consultoría e ingeniería medioambiental y para la gestión de los recursos naturales

Residuos: fabricación de contenedores, equipos para el tratamiento y vehículos para transporte y

almacenamiento; servicios de recogida, tratamiento e incineración; producción de bolsas de basura

Aguas residuales: ingeniería, servicios y construcción de sistemas de recogida y tratamiento; fabricación de depuradoras, reactivos, fosas sépticas; equipos y tuberías para conducción, recogida y tratamiento

Fuente: Instituto Nacional de Estadística (2013).

otorgadas por entidades públicas o privadas para la realización de actividades de protección ambiental, así como el importe de los trabajos realizados por la entidad para la construcción, instalación o montaje de su inmovilizado ambiental, si se siguiese un modelo contable dualista ${ }^{5}$.

En este contexto, procede cuestionarse si cualquier venta de bienes (por ejemplo, material de construcción) o prestación de servicios (por ejemplo, el asesoramiento de un ingeniero) tendría la calificación de ambientalmente positivos para las entidades proveedoras si su destino en la entidad adquirente fuese ser transformados en bienes o servicios ambientales para uso interno (por ejemplo, la construcción de una depuradora para las aguas residuales vertidas por la propia empresa) o para su comercialización a terceros (por ejemplo, la venta llave en mano de la citada depuradora). Parece razonable sostener que si tales bienes y servicios no tenían a priori la calificación de ambientales, y dado que el acto de la venta implica la cesión de los riesgos y beneficios asociados a dichos elementos y, por tanto, la transferencia del control de los mismos al comprador,

\footnotetext{
${ }^{5}$ Los modelos contables dualistas se caracterizan por la existencia de 2 ámbitos claramente definidos en la entidad, denominados ámbito externo e interno, que son independientes entre sí y que conforman las denominadas contabilidad externa o financiera e interna o de costos, respectivamente. Esta independencia provoca que los bienes y servicios fabricados por la entidad en su ámbito interno, con sus propios medios humanos y materiales, no puedan ser reconocidos en el balance de la contabilidad financiera salvo que se recurra a su activación o afloramiento mediante una cuenta de ingresos, como es el caso de los trabajos realizados para el inmovilizado.
} 
el vendedor no debe reconocer ingresos ambientales porque, en este caso, su finalidad última no está predeterminada por las características del bien vendido o servicio prestado. Es decir, la venta de cemento no origina un ingreso ambiental porque es susceptible de usos diversos, no estando asociado a un fin concreto, como pudiera ser el ambiental. Sin embargo, la venta de un filtro para una instalación de desulfuración sí podría traducirse en un ingreso ambiental positivo porque su fin, y no otro, es el de ser utilizado en una maquinaria destinada a minimizar los impactos ambientales derivados de las emisiones de azufre.

Si la entidad obtiene ingresos considerados ambientalmente positivos, la discriminación realizada en el apartado precedente entre gastos ambientales positivos y otros gastos podría ser susceptible de aplicación a este caso. Así, podría diferenciarse entre ingresos ambientalmente positivos (Ip), trabajos realizados para el inmovilizado ambiental (Itia $)^{6}$ y otros ingresos (Io), como se indica a continuación:

$$
I=I p+I t i a+I o
$$

Teniendo en cuenta lo expresado en (10), la expresión analítica del resultado podría representarse de la siguiente forma:

$$
R=(I p+I t i a+I o)-(G p+G i r+G i s+G o)
$$

La expresión (12) podría ser dividida o desglosada en 2: una que represente el resultado ambiental positivo $(R p)$, integrado por los ingresos ambientalmente positivos y los gastos que han sido incurridos para obtener dichos ingresos, y otra que refleje el resultado formado por los demás ingresos de la entidad menos los gastos realizados para obtener dichos ingresos $(R o)$ :

$$
R=R p+R o
$$

Ahora bien, en atención al funcionamiento operativo de la empresa, es obvia la existencia de gastos que, estando destinados a la obtención de ingresos derivados de la venta de bienes y prestaciones de servicios ambientalmente positivos (por ejemplo, los servicios de consultoría ambiental), carecen de tal carácter (por ejemplo, consumo de electricidad) porque no están destinados a la prevención, corrección o minimización de los impactos causados por la propia empresa. Aunque determinados gastos no respondan a transacciones que produzcan por sí mismas impactos positivos significativos sobre el medio ambiente, pueden ser incurridos para obtener productos y servicios cuya colocación en el mercado suponga un efecto ambiental positivo relevante. Asimismo, pueden existir gastos de signo ambientalmente positivo (por ejemplo, la amortización de una depuradora) que son imputados a la generación de ingresos por la venta de bienes o prestaciones de servicios que no acarrean un impacto positivo relevante sobre el entorno (por ejemplo, el alojamiento hotelero). Tanto los unos como los otros pueden ser objeto de una adecuada identificación.

Ello lleva a distinguir, para cada categoría de gastos, entre los que serán destinados a la venta de bienes y servicios positivos para el medio ambiente y la manufactura de activos propios destinados a la protección ambiental, y los que serán aplicados a la obtención de otros ingresos ${ }^{7}$.

\footnotetext{
${ }^{6}$ El tratamiento separado de los trabajos realizados para el inmovilizado ambiental responde a la conveniencia de distinguirlos de las transacciones con otras entidades y de facilitar la comparación con empresas que utilicen un sistema contable monista.

${ }^{7}$ Nótese que, de acuerdo con el enfoque adoptado en el presente trabajo, no cabría la existencia de otros gastos de la entidad que fuesen destinados a la construcción de activos ambientales propios, pues de la propia conceptuación de gasto
} 
Las desagregaciones a realizar serían las siguientes, donde el sufijo «ia» indica que el gasto se aplica a la generación de ingresos ambientales y el sufijo «oi» que el gasto está relacionado con la obtención de otros ingresos:

$$
\begin{aligned}
& G p=\text { Gpia }+ \text { Gpoi } \\
& \text { Gir }=\text { Giria }+ \text { Giroi } \\
& \text { Gis }=\text { Gisia }+ \text { Gisoi } \\
& \text { Go }=\text { Goia }+ \text { Gooi }
\end{aligned}
$$

De esta forma, la expresión (13) quedaría como se indica a continuación:

$$
\begin{aligned}
& R=R p+R o= \\
& (I p+I t i a+I o)-[(G p i a+\text { Giria }+ \text { Gisia }+ \text { Goia })+(\text { Gpoi }+ \text { Giroi }+ \text { Gisoi }+ \text { Gooi })]
\end{aligned}
$$

donde:

$$
\begin{aligned}
& R p=(I p+I t i a)-(\text { Gpia }+ \text { Giria }+ \text { Gisia }+ \text { Goia }) \\
& R o=I o-(\text { Gpoi }+ \text { Giroi }+ \text { Gisoi }+ \text { Gooi })
\end{aligned}
$$

Según estas expresiones, el resultado ambiental positivo es considerado como una segmentación del resultado contable de acuerdo con las líneas de productos de la empresa, por lo que más bien cabría denominarlo «resultado del negocio ambiental» (si se excluyen los trabajos realizados para el inmovilizado ambiental), dada la visible omisión en su cómputo de determinados gastos ambientales que, en virtud del Principio de correlación de ingresos y gastos, han sido imputados al resto de los ingresos de la entidad ${ }^{8}$.

Las magnitudes obtenidas constituyen una etapa más avanzada en el proceso de discriminación de las actividades ambientales de la empresa, permitiendo a los grupos de interés conocer de forma más detallada los aspectos positivos de la gestión ambiental. Sin embargo, es razonable cuestionarse el hecho de que solo gocen de visibilidad los gastos e ingresos ambientalmente positivos, mientras los de carácter negativo, es decir, aquellos que constituyen presiones sobre el estado del medio ambiente, permanezcan ocultos entre el resto de las magnitudes constitutivas del resultado.

\section{Un paso más: haciendo visibles los ingresos y gastos ambientalmente negativos}

Al objeto de alcanzar un mayor conocimiento acerca de la realidad de las operaciones ambientales de la empresa, cabría diferenciar, dentro de las restantes magnitudes económicas formativas del resultado, entre aquellas que no reportan efecto alguno en el medio ambiente (neutrales) y aquellas otras cuya ocurrencia supone un efecto adverso directo o indirecto, mediato o inmediato, cierto o probable sobre el estado del medio ambiente, conformando estas últimas las transacciones ambientalmente negativas.

\footnotetext{
ambiental, cualquier gasto que sea incurrido para manufacturar un activo destinado a la protección ambiental de la entidad tiene la consideración propia de gasto ambiental.

${ }^{8}$ Precisamente, el Principio de correlación de ingresos y gastos impide la construcción de una magnitud resuntiva de todos los ingresos y gastos ambientales del ejercicio, que pudiera titularse como«resultado ambiental total» o similar, al no existir una vinculación directa o indirecta entre los ingresos ambientales generados y los gastos incurridos para obtener otros ingresos.
} 
Aunque la evidencia existente al respecto es controvertida (Ingram, 1978; Freedman y Jaggi, 1986; Jaggi y Freedman, 1992; Blacconiere y Patten, 1994; Little, Muoghalu y Robinson, 1995; Klassen y Mclaughlin, 1996; entre otros), en principio, la correcta identificación, medida y valoración de dichas transacciones permitiría un conocimiento más ajustado de la actividad realizada por la empresa en el campo ambiental y, por extensión, de su grado de compromiso con la sociedad en favor de la protección de la salud, la propiedad ajena y el medio ambiente en general.

No obstante, no puede obviarse el carácter polémico de definir algo como «negativo» para el medio ambiente, por los problemas de deslegitimación a que podría verse sometida la empresa. Cabe exponer en este sentido, y a modo de ejemplo, el debate suscitado para la reforma del FTSE4Good $^{9}$, al objeto de que la producción de energía nuclear no fuese un obstáculo para formar parte del índice, con base en el argumento de que dicha fuente de energía se considera como una opción válida para mitigar el cambio climático, por sus prácticamente nulas emisiones de dióxido de carbono (Iberdrola, 2007), como finalmente se acabaría aceptando en 2010.

De acuerdo con la teoría de la revelación voluntaria (Bewley y Li, 2000; Clarkson, Li, Richardson y Vasvari, 2008; Cho, Roberts y Patten, 2012), la empresa debería dar cuenta en sus informes tanto de los aspectos positivos derivados de su desempeño como de los negativos. No obstante, es factible que la empresa omita aquellos aspectos que pudieran afectar a su posición en el mercado y en la sociedad. En tal caso, dicho informe actuaría como un instrumento para legitimar la gestión empresarial (Guthrie y Parker, 1989; Deegan y Rankin, 1997). Así, según la teoría de la legitimidad, los informes corporativos serían un medio a través del cual mejorar la imagen percibida por la sociedad en respuesta a la presión de los grupos de interés relevantes y «legitimarse» ante ellos (Lindblom, 1994; Gray, Owen y Adams, 1996; Deegan, 2002; Gumb, 2007). Según esta visión, las empresas con un peor desempeño tendrán un estímulo para difundir información positiva y ocultar la negativa al objeto de minimizar potenciales costos derivados de su confrontación con los grupos de interés (por ejemplo, el gobierno).

A fin de simplificar el desarrollo analítico propuesto, se desagregará de la magnitud representativa del resto de los gastos de la entidad (Go) los gastos ambientalmente negativos (Gn), es decir, aquellos que reflejan transacciones asociadas a impactos adversos sobre el entorno. Considerando la distinción entre magnitudes ambientalmente positivas, negativas y otras, los gastos de la entidad quedarían representados de la siguiente forma:

$$
G=G p+G i r+G i s+G n+G o
$$

Entre los gastos susceptibles de consideración como ambientalmente negativos cabría citar, a título ilustrativo, los relacionados con la adquisición de recursos naturales, materias primas no renovables/críticas, materias primas vitales para la economía nacional, materiales tóxicos y peligrosos, materiales cuyos proveedores posean un historial ambiental negativo, así como los gastos de transporte de aprovisionamientos y suministros.

La traslación al capítulo de ingresos de la distinción entre transacciones ambientalmente neutrales y ambientalmente negativas desemboca en la consiguiente desagregación de las magnitudes alusivas al ingreso, en el supuesto de que la entidad fabricase bienes destinados a la realización de

\footnotetext{
${ }^{9}$ La serie de índices FTSE4Good ha sido diseñada para medir objetivamente el desempeño de las empresas que cumplen con las normas reconocidas a nivel mundial de responsabilidad corporativa. La gestión transparente y los criterios de FTSE4Good son utilizados por consultores, propietarios de activos, gestores de fondos, bancos de inversión, bolsas de valores y corredores a la hora de evaluar o crear productos de inversión responsable. Más información en: http://www.ftse.com/Indices/FTSE4Good_Index_Series/index.jsp
} 
actividades con impactos de diferente signo sobre el medio ambiente. Así, la distinción entre ingresos ambientales positivos (Ip), trabajos realizados para el inmovilizado ambiental (Itia), ingresos ambientales negativos (In) y otros (Io), daría lugar a la siguiente expresión representativa del ingreso:

$$
I=I p+I t i a+I n+I o
$$

Bajo la calificación de ingresos ambientales negativos se incluirían los ocasionados por la venta de bienes y prestaciones de servicios que ocasionasen directa o indirectamente impactos perjudiciales en el entorno, como consecuencia de su normal aplicación por los usuarios, considerando las especificaciones técnicas del fabricante. A modo de ejemplo cabría considerar la venta de recursos naturales críticos, productos elaborados con materias primas no renovables o vitales, así como de productos tóxicos y peligrosos.

Como consecuencia de (17) y (18), la expresión analítica del resultado podría representarse de la forma que se describe a continuación, en la que se realiza una desagregación de las magnitudes ambientales atendiendo al vínculo positivo, negativo o neutro existente con el medio ambiente:

$$
R=(I p+I t i a+I n+I o)-(G p+G i r+G i s+G n+G o)
$$

Cabría segmentar las magnitudes reflejadas en (19), distinguiendo las positivas, que conformarían el resultado del negocio ambientalmente positivo $(R p)$, de las negativas, que darían lugar al resultado del negocio ambiental negativo $(R n)$. El resto de las transacciones que carezcan de impacto significativo sobre el medio ambiente conformarían el resultado no ambiental $(\mathrm{Ro})$ :

$$
R=R p+R n+R o
$$

Ahora bien, análogamente a lo expresado en (15), existen gastos ambientalmente negativos que pueden ser dedicados tanto a la obtención de ingresos positivos para el medio ambiente como negativos, o incluso neutros, y viceversa. A modo de ejemplo, y para el primer caso, cabe citar el consumo de combustible fósil para el arranque de un aerogenerador de gran potencia; para el segundo, el transporte por carretera de las ventas de productos tóxicos y peligrosos; o finalmente, el consumo de electricidad procedente de fuentes no renovables en la prestación de servicios financieros. Así, cada una de las magnitudes de gasto consideradas se desglosaría en gastos destinados a la obtención de ingresos ambientales positivos, negativos y neutros, según fuera el caso. Las equivalencias a realizar serían las siguientes:

$$
\begin{aligned}
& \text { Gp }=\text { Gpia + Gpin + Gpio } \\
& \text { Gir }=\text { Giria + Girin + Girio } \\
& \text { Gis }=\text { Gisia + Gisin + Gisio } \\
& \text { Gn }=\text { Gnia + Gnin + Gnio } \\
& \text { Go }=\text { Goia + Goin + Goio }
\end{aligned}
$$

donde el sufijo «ia» indica que el gasto se aplica a la generación de ingresos positivos, el sufijo «in» que el gasto se incurre en la obtención de ingresos negativos, y el sufijo «io» que el gasto está relacionado con la realización de otros ingresos. La expresión (20) quedaría como sigue:

$$
\begin{aligned}
& R=\mathrm{Rp}+\mathrm{Rn}+\mathrm{Ro}=(\mathrm{Ip}+\mathrm{Itia}+\mathrm{In}+\mathrm{Io})- \\
& {[(\text { Gpia }+ \text { Giria }+ \text { Gisia }+ \text { Gnia }+ \text { Goia })+(\text { Gpin }+ \text { Girin }+ \text { Gisin }+ \text { Gnin }+ \text { Goin })+} \\
& (\text { Gpio + Girio + Gisio + Gnio + Goio })]
\end{aligned}
$$




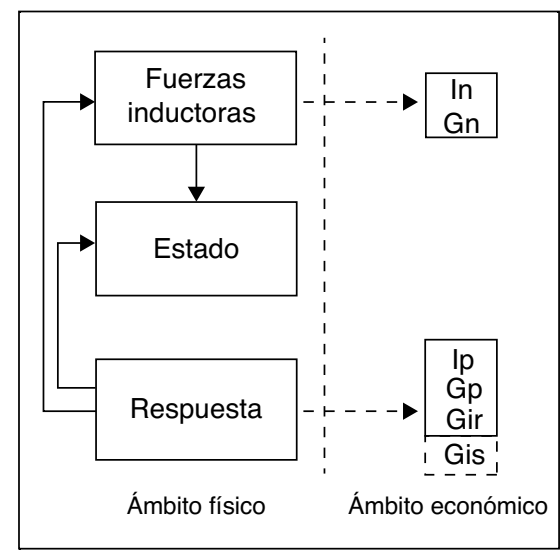

Figura 2. El modelo Fuerzas inductoras-Estado-Respuesta con ingresos y gastos ambientalmente positivos y negativos. Fuente: Elaboración propia.

donde:

$$
\begin{aligned}
& \mathrm{Rp}=(\mathrm{Ip}+\mathrm{Itia})-(\text { Gpia }+ \text { Giria }+ \text { Gisia }+ \text { Gnia }+ \text { Goia }) \\
& \mathrm{Rn}=\mathrm{In}-(\text { Gpin }+ \text { Girin }+ \text { Gisin }+ \text { Gnin }+ \text { Goin }) \\
& \mathrm{Ro}=\mathrm{Io}-(\text { Gpio }+ \text { Girio }+ \text { Gisio }+ \text { Gnio }+ \text { Goio })
\end{aligned}
$$

Los diferentes tipos de resultado calculados responden a una segmentación del resultado contable de acuerdo con las líneas de productos de la empresa (si no se consideran los trabajos realizados para el inmovilizado ambiental), atendiendo al Principio de correlación de ingresos y gastos. Como se observa, tal discriminación en modo alguno implica una independencia total y absoluta de las diferentes clases de magnitudes consideradas (ambientales positivas, ambientales negativas y no ambientales), toda vez que, salvo casos muy particulares, pueden existir correlaciones entre magnitudes de distinto signo, de forma tal que un ingreso considerado ambientalmente positivo puede tener asociado un gasto ambientalmente negativo o uno de carácter neutro, y viceversa. Incluso, un gasto ambientalmente positivo (por ejemplo, el asociado a la reparación de un daño ambiental) puede tener su origen en otro gasto ambientalmente negativo (tal como la amortización de una maquinaria contaminante). Asimismo, puede generarse un gasto ambientalmente negativo para dar cumplimiento a un objetivo ambiental (por ejemplo, el transporte por carretera de residuos industriales). Todo ello se traduce en la necesidad de acometer el pertinente análisis del ciclo vital de la producción de la empresa.

Desde la perspectiva de los modelos Presión-Estado-Respuesta comentados anteriormente, cabría asimilar los ingresos y gastos de transacciones positivas para el medio ambiente al valor económico de las medidas de respuesta de la entidad ante las presiones ambientales asociadas a los gastos e ingresos ambientalmente negativos y los daños causados en el estado del medio ambiente, bien por la propia entidad, bien por terceros, tal y como se presenta en la figura 2.

\section{Conclusiones}

La noción de resultado ambiental enunciada a lo largo del presente trabajo se apoya en la agregación de un conjunto de magnitudes ya reconocidas convencionalmente por el sistema de información contable de la entidad. Es decir, se basa en una discriminación de ingresos y gastos 
que ya estaban registrados por la empresa a los que, «simplemente», se les han añadido unas etiquetas clasificativas.

Aunque pudiera parecer que la única aportación metodológica ha consistido en la discriminación de las transacciones habitualmente reconocidas por la empresa entre operaciones no ambientales y ambientales, y, dentro de estas, entre positivas y negativas para el entorno, tal categorización constituye una aportación al diseño de sistemas de información ambiental corporativos. En este sentido, se propone la identificación de todas las transacciones realizadas por la empresa, no solo de aquellas con efectos potencial o efectivamente nocivos para el medio ambiente, sino también de las ambientalmente favorables. De esta forma, se podrían delimitar con mayor precisión los riesgos corporativos, amén de facilitar la medición de los esfuerzos realizados a favor del medio ambiente, pudiendo, incluso, establecerse como un objetivo la reducción de todas las transacciones ambientalmente negativas y su sustitución por operaciones que tengan un impacto neutral o positivo sobre el entorno, previa ponderación de sus efectos sobre el patrimonio, la situación financiera y los resultados de la compañía.

No obstante lo anterior, esta propuesta adolece de algunas limitaciones. En este sentido, un primer problema es que la empresa, por ignorancia, omisión o indiferencia, no tenga identificados sus gastos e ingresos ambientales. A ello contribuye el hecho de que no exista una clasificación normalizada para establecer qué actividades son ambientales y cuáles no, con el riesgo de que la empresa defina como tales partidas que técnica o conceptualmente no procediesen, como es el caso de las relacionadas con la seguridad e higiene laboral, la política de relaciones públicas o la repoblación forestal con fines comerciales. También es posible que la empresa, condicionada por la normativa tributaria, considere como ambientales solo aquellas partidas que sean susceptibles de acogerse a las ayudas o incentivos fiscales establecidos por los poderes públicos y deje de identificar el resto de los conceptos.

Otro aspecto a efectos de control de gestión es que una reducción de los gastos ambientales positivos de un año al siguiente puede ser atribuida a una pérdida de interés o una relajación de los estándares ambientales de la empresa, cuando realmente obedecen a que la empresa dedicó el año anterior parte de sus recursos a la autoconstrucción de activos no corrientes de protección ambientales, por lo que debido a la técnica de reconocimiento contable del modelo dualista figuraron como mayores gastos del ejercicio. En teoría, para solventar esta discrepancia debería verificarse que se ha reconocido como contrapartida una cuenta de ingreso en concepto de trabajos realizados para el inmovilizado. En cualquier caso, la adquisición o autoconstrucción de un activo ambiental debería traducirse en un incremento de las amortizaciones y los gastos de operación y mantenimiento que, al estar afectos a actividades de protección ambiental, deberían reconocerse como tales. A ello se suma que posibles cambios normativos influyan en la definición de lo que es o no es gasto ambiental, por lo que se hace necesario revelar y mostrar los efectos de las nuevas definiciones del concepto.

Por otra parte, es factible que la empresa incurra en unos desembolsos espectaculares en favor del medio ambiente como consecuencia, precisamente, de la naturaleza altamente contaminante de la actividad desarrollada y, como tales, tengan la consideración de ambientalmente positivos. Sin embargo, la presunta mejora ambiental que conlleven puede ser muy inferior a la que se conseguiría, bajo otras circunstancias, con una menor inversión, derivando en la necesidad de estudiar la eficiencia ambiental de la empresa. Para ello debe acometerse la vinculación de tales magnitudes a los objetivos ambientales perseguidos mediante su comparación con mediciones físicas de hechos ambientales, como la emisión de residuos o el consumo de energía. Ello implicaría recurrir a instrumentos de representación alternativos, como los indicadores de gestión ambiental, en el marco de, por ejemplo, un modelo causal como el Presión-Estado-Respuesta, a fin de calibrar 
adecuadamente la importancia que sobre el medio ambiente tienen los impactos originados por la realización de la actividad considerada.

Es obvio que este enfoque plantea claros problemas de homogeneización, en el supuesto caso de que se desease obtener una cifra única omnicomprensiva de la gestión realizada, siendo necesario establecer un factor de ponderación que haga equivalentes cada uno de los objetivos propuestos.

Sumado a lo anterior, el establecimiento de modelos que guíen hacia la total transparencia puede provocar efectos opuestos a los perseguidos. Así, considerar la existencia de magnitudes ambientalmente positivas y negativas podría llevar a la dirección de la compañía a plantear la búsqueda de soluciones ineficientes desde el punto de vista social, aunque sí eficientes desde la imagen corporativa, salvando su responsabilidad pública frente al daño ambiental mediante el blanqueo de cuentas ambientales. En efecto, una empresa puede disminuir sus emisiones atmosféricas simplemente sustituyendo la maquinaria accionada gracias a combustibles fósiles por otra basada en la electricidad, provocando, no obstante, que la compañía eléctrica suministradora incremente sus emisiones. También se podría recurrir a la constitución de empresas dedicadas a la gestión de las actividades conflictivas mediante terceros interpuestos o en otros países con una legislación ambiental más laxa. De ahí la importancia de que los grupos de interés relevantes sean capaces de identificar todas las transacciones potencial o efectivamente causantes de impactos ambientales, sean estos directa o indirectamente responsabilidad de la empresa.

En atención a lo expuesto, una extensión lógica del presente trabajo se encuentra en la realización de un estudio de campo con empresas de sectores con interacciones significativas con su medio ambiente al objeto de evaluar las limitaciones planteadas y proponer cursos de acción alternativos que contribuyan a solventar las mismas.

\section{Referencias}

Asociación Española de Normalización y Certificación. (2005). UNE 50011: 2005 Gestión ambiental. Guía para la evaluación de los costes ambientales. Costes ambientales internos. Madrid: AENOR.

Atkinson, G. (2000). Measuring corporate sustainability. Journal of Environmental Planning and Management, 43(2), 232-252.

Belkaoui, A. (1995). Accounting theory (3rd ed.). London: Quorum Books.

Bennett, M. y James, P. (1997). Environmental-related management accounting in North America and its implications for UK companies. En C. Hibbitt y H. Blokdjik (Eds.), Environmental accounting and sustainable development: The final report. Amsterdam: Limperg Instituut.

Bewley, K. y Li, Y. (2000). Disclosure of environmental information by Canadian manufacturing companies: A voluntary disclosure perspective. Advances in Environmental Accounting and Management, 1, 201-226.

Blacconiere, W. G. y Patten, D. M. (1994). Environmental disclosures, regulatory costs, and changes in firm value. Journal of Accounting and Economics, 18, 355-377.

Cho, C. H., Roberts, R. W. y Patten, D. M. (2010). The language of U. S. corporate environmental disclosure. Accounting, Organizations and Society, 35(4), 431-443.

Cho, C. H., Roberts, R. W. y Patten, D. M. (2012). Corporate disclosure of environmental capital expenditures: A test of alternative theories. Accounting, Auditing \& Accountability Journal, 25(3), 486-507.

Clarkson, P. M., Li, Y., Richardson, G. D. y Vasvari, F. P. (2008). Revisiting the relation between environmental performance and environmental disclosure: An empirical analysis. Accounting, Organizations and Society, 33(4), 303-327.

Comisión Europea. (2001). Recomendación de la Comisión, de 30 de mayo de 2001, relativa al reconocimiento, la medición y la publicación de las cuestiones medioambientales en las cuentas anuales y los informes anuales de las empresas (2001/453/CE). Diario Oficial de las Comunidades Europeas.

Deegan, C. (2002). The legitimizing effect of social and environmental disclosures: A theoretical foundation. Accounting, Auditing \& Accountability Journal, 15(3), 282-311.

Deegan, C. y Rankin, M. (1997). The materiality of environmental information to users of annual reports. Accounting, Auditing \& Accountability Journal, 10(4), 562-583. 
Déniz Mayor, J. J. y Verona Martel, M. C. (2012). Modelos causales de indicadores en la información corporativa sobre sostenibilidad. Criterio Libre, 10(16), 69-88.

Eurostat. (2000). Classification of environmental protection activities and expenditure. Luxembourg: Office for Official Publications of the European Communities.

Eurostat. (2007). Environmental expenditure statistics. General government and specialised producers data collection handbook. Luxembourg: Office for Official Publications of the European Communities.

Eurostat. (2009). The environmental goods and services sector. A data collection handbook. Luxembourg: Office for Official Publications of the European Communities.

Freedman, M. y Jaggi, B. (1986). Risk evaluation of firms disclosing pollution information in financial statements. Advances in Accounting, 3, 113-125.

Friend, A. y Rapport, D. (1979). Towards a comprehensive framework for environment statistics: A stress-response approach. Ottawa: Statistics Canada.

Gladwin, T., Kennelly, J. y Krause, T. (1995). Shifting paradigms for sustainable development: Implications for management theory and research. Academy of Management Review, 20(4), 874-907.

Gray, R., Bebbington, J. y Walters, D. (1993). Accounting for the environment. London: Paul Chapman.

Gray, R., Owen, D. y Adams, C. (1996). Accounting \& accountability: Changes and challenges in corporate social and environmental reporting. Harlow: Prentice Hall.

Greider, W. (1997). One world, ready or not. New York: Simon and Schuster.

Gumb, B. (2007). What is shown, what is hidden: Compulsory disclosure as a spectacle. Critical Perspectives on Accounting, 18(7), 807-828.

Guthrie, J. y Parker, L. D. (1989). Corporate social reporting: A rebuttal of legitimacy theory. Accounting and Business Research, 19(76), 343-352.

Guttman Sterimberg, E., Zorro Sánchez, C., Cuervo de Forero, A. y Ramírez, J. C. (2004). Diseño de un sistema de indicadores socio ambientales para el Distrito Capital de Bogotá. CEPAL/PNUD COL/01/002. Bogotá: CEPAL Naciones Unidas.

Iberdrola (2007). Informe de sostenibilidad 2007. Preguntas del Comité Independiente de Expertos respecto al Informe del ejercicio 2006 [consultado 30 Mar 2013]. Disponible en: www.iberdrola.es/webibd/gc/prod/es/doc/ Preguntas_comite.pdf

Ingram, R. W. (1978). An investigation of the information content of (certain) social responsibility disclosure. Journal of Accounting Research, 16(2), 270-285.

Instituto de Contabilidad y Auditoría de Cuentas (2002). Resolución de 25 de marzo de 2002, del Instituto de Contabilidad y Auditoría de Cuentas, por la que se aprueban normas para el reconocimiento, valoración e información de los aspectos medioambientales en las cuentas anuales. BOE núm. 81, de 4 de abril de 2002. pp. 12831-4 [consultado 30 Mar 2013 ]. Disponible en: http://www.boe.es/diario_boe/txt.php?id=BOE-A-2002-6389

Instituto Nacional de Estadística. (2013). Encuesta sobre medio ambiente en la industria 2011. Madrid: INE.

International Accounting Standards Board. (2003). IAS 16. Property, plant and equipment. London: IASB.

International Accounting Standards Committee. (1998). IAS 37. Provisions, contingent liabilities and contingent assets. London: IASC.

Jaggi, B. y Freedman, M. (1992). An examination of the impact of pollution performance on economic and market performance: Pulp and paper firms. Journal of Business Finance and Accounting, 19(5), 697-714.

Jaszi, G. (1973). Comments on F. Thomas Juster's “A Framework for the Measurement of Social and Economic Performance". In Studies in Income and Wealth, Vol. 28. New York: National Bureau of Economic Research.

Klassen, R. D. y Mclaughlin, C. P. (1996). The impact of environmental management on firm performance. Management Science, 42(8), 1199-1214.

Leonard, H. J. (1988). Pollution and the struggle for a world product: Multinational corporations, environment, and the struggle for international comparative advantage. Cambridge: Cambridge University Press.

Lindblom, C.K. (1994). The implications of organizational legitimacy for corporate social performance and disclosure. Critical Perspectives on Accounting Conference, New York.

Little, P., Muoghalu, M. I. y Robinson, H. D. (1995). Hazardous waste lawsuits, financial disclosure, and investors' interests. Journal of Accounting, Auditing and Finance, 10(2), 383-400.

Martínez Alier, J. (1992). Obstáculos distributivos contra la política ambiental internacional: los fracasos de Río de Janeiro y perspectivas después de Río. Información Comercial Española., 711, 87-106.

Michael, B. (2003). Corporate social responsibility in international development: An overview and critique. Corporate Social Responsibility and Environmental Management, 10(3), 115-128.

Moneva, J. M., Archel, P. y Correa, C. (2006). GRI and the camouflaging of corporate unsustainability. Accounting Forum, 30(2), 121-137. 
Mortensen, L. (1997). The Driving Force-State-Response framework used by the UNCSD. En S. Billharz y B. Moldan (Eds.), Sustainability indicators: A report on the Project on Indicators of Sustainable Development. West Sussex: John Wiley \& Sons.

Newell, P. (2005). Citizenship, accountability and community: The limits of the CSR agenda. International Affairs, 81(3), 541-557.

Organisation for Economic Co-operation and Development (1993). OECD core set of indicators for environmental performance reviews, Environment Monographs, No. 83. OECD/GD (93) 179. Paris: OECD.

Organisation for Economic Co-operation and Development (1997). OECD environmental performance reviews. A practical introduction. OECD/GD (97) 35. Paris: OECD.

Organisation for Economic Co-operation and Development (2003). OECD environmental indicators-Development, measurement and use. Reference paper. Paris: OECD.

Organisation for Economic Co-operation Development/Eurostat. (1999). The environmental goods and services industry: Manual for data collection and analysis. Paris: OECD.

Quiroga Martínez, R. (2007). Indicadores ambientales y de desarrollo sostenible: avances y perspectivas para América Latina y el Caribe. Santiago de Chile: CEPAL.

Silverstein, M. (1989). The environmental factor. Its impact on the future of the world economy and your investments. Chicago: Dearborn Financial Publishing.

Stewart, R. (1993). Environmental regulation and international competitiveness. Yale Law Journal, 102, $2039-2106$.

United Nations (1995). General Discussion of progress in the implementation of Agenda 21, focusing on the crosssectorial Components of Agenda 21 and the critical element of sustainability. E/CN.17/1995/18 (annexes), 24 March. New York: United Nations Commission on Sustainable Development [consultado 30 Mar 2013]. Disponible en: http://www.un.org/earthwatch/about/docs/csd3sgan.htm

United States Environmental Protection Agency. (1995). A conceptual framework to support development and use of environmental information in decision-making. EPA 239-R-95-012. Washington D.C.: USEPA. 